PHYSICAL REVIEW D 96, 129901(E) (2017)

\title{
Erratum: On-shell effective field theory: A systematic tool \\ to compute power corrections to the hard thermal loops \\ [Phys. Rev. D 94, 025017 (2016)]
}

Cristina Manuel, Joan Soto, and Stephan Stetina

(Received 27 November 2017; published 15 December 2017)

DOI: 10.1103/PhysRevD.96.129901

We have found the following typographical and numerical errors, which affect only the transverse polarization tensors.

Eq. (15): In the first matrix, there is a global sign missing in the second row, second column entry.

Eq. (55): There is a global factor $1 / 2$ missing in the rhs of the equation.

Eq. (66): A global factor $1 /(2+\epsilon)$ is missing in the lhs of the equation.

Eq. (75): A global factor $1 /(2+\epsilon)$ is missing in the lhs of the equation.

Eq. (79): This equation should read as follows:

$$
\Pi_{\text {total,(3) }}^{T}\left(l_{0}, \mathbf{l}\right)=\frac{\alpha}{\pi}\left[\frac{1}{2} l_{0}^{2}-\frac{2}{3} \mathbf{l}^{2}+\frac{1}{6} \frac{l_{0}^{4}}{\mathbf{l}^{2}}-\frac{1}{12} \frac{l_{0}^{3}}{|\mathbf{I}|^{3}}\left(l_{0}^{2}+2 \mathbf{l}^{2}-3 \frac{\mathbf{l}^{4}}{l_{0}^{2}}\right) \times\left(\ln \left|\frac{l_{0}+|\mathbf{I}|}{l_{0}-|\mathbf{I}|}\right|-i \pi \Theta\left(|\mathbf{I}|^{2}-l_{0}^{2}\right)\right)\right] .
$$

Eq. (D8): A factor $e^{2}$ is missing in the rhs, and indices in one term should be changed to $v^{j} l^{i} \rightarrow v^{i} l^{j}$. The conclusions of the paper remain unchanged. 